\title{
Detection of SARS-CoV-2 in placental but not fetal tissues in the second trimester
}



Received: 22 September 2020 / Revised: 6 October 2020 / Accepted: 12 November 2020 / Published online: 30 November 2020

(c) Springer Nature America, Inc. 2020

\section{To the Editor:}

The data on placental and fetal involvement in severe acute respiratory syndrome coronavirus 2 (SARS-CoV-2) infection during pregnancy are limited. We analyzed placental and fetal tissues for the presence of SARS-CoV-2 in two infected women who presented with a miscarriage and a preterm labor in the second trimester.

The study was approved by the Institutional Review Board. The tissues were studied by immunohistochemistry (IHC) using antibodies against SARS-CoV-2 spike (clone 1A9; GeneTex, Irvine, CA) and nucleocapsid (clone 0001; Sino Biological, Wayne, PA) proteins and in-situ hybridization (ISH) using the RNAscope-ProbeV-nCoV2019-S (Advanced-Cell-Diagnostics, Hayward, CA). Quantitative reverse transcription polymerase chain reaction (qRT-PCR) was carried out using RNA extracted from formalin-fixed paraffin embedded tissues and SARS-CoV-2 primer/probe sets (Integrated DNA Technology, Coralville, Iowa). Each assay included a standard curve, using the 2019nCoV_N_Positive Control (Integrated DNA Technology, Coralville, Iowa).

Both women tested positive for SARS-CoV-2 by the nasopharyngeal swab test, but were asymptomatic for COVID-19 before, during or within 14 days after delivery. A 17 year-old gravida 1 para 0 presented with preterm

Supplementary information The online version of this article (https:// doi.org/10.1038/s41372-020-00877-8) contains supplementary material, which is available to authorized users.

$\checkmark$ Larisa Debelenko

1d2863@cumc.columbia.edu

1 Department of Pathology and Cell Biology, Columbia University, New York, NY, USA

2 Department of Medicine, Columbia University, New York, NY, USA premature rupture of membranes at $18+$ weeks and delivered a non-viable female fetus. A 29 year-old gravida 1 para 0 presented in labor at $23+$ weeks and delivered a male neonate with Apgar scores 2, 2, and 2. A cardiac arrest was registered on $8 \mathrm{~min}$ of life and resuscitation was unsuccessful. The neonatal nasal swab test for SARS-CoV-2 was negative.

Placental and fetal pathologies as well as results of the tissue viral studies are presented in Table 1 . SARS-CoV-2 qRT-PCR was positive in placentas and negative in fetal organs in both cases. Neither placentas, nor fetal organs stained for the virus by IHC and ISH. This discrepancy can be explained by a much lower sensitivity of IHC and ISH, comparing to RT-PCR. To date, we found at least ten reported cases of SARS-CoV-2 detected in the placental tissue by RT-PCR [1-5] and only in five cases was the virus demonstrated by IHC and/or ISH $[2,3]$.

Lack of viral sequences in umbilical cords and fetal organs argues against a vertical transmission of SARS$\mathrm{CoV}-2$, while the presence of placental and fetal pathologies previously established to be associated with fetal demise can explain the abortion and the preterm labor. In the 1st case, the placenta showed chronic deciduitis (Supplementary Fig. 1), which is known to be associated with an abnormal immune response to pregnancy and spontaneous abortions with normal karyotype. In the 2nd case, placental and neonatal pulmonary pathologies provided a convincing evidence of involvement by Group B Streptococcus agalactiae (Supplementaty Fig. 2), a known cause of premature labor and neonatal death.

We demonstrated SARS-CoV-2 involvement of placentas detectable by qRT-PCR in asymptomatic COVID19-infected pregnant women in the second trimester. Our data are in accord with published reports on the low incidence of vertical transmission of the virus. However, more data are needed to determine the overall feto-maternal risks of SARS-CoV-2 infection at different terms of gestation. 


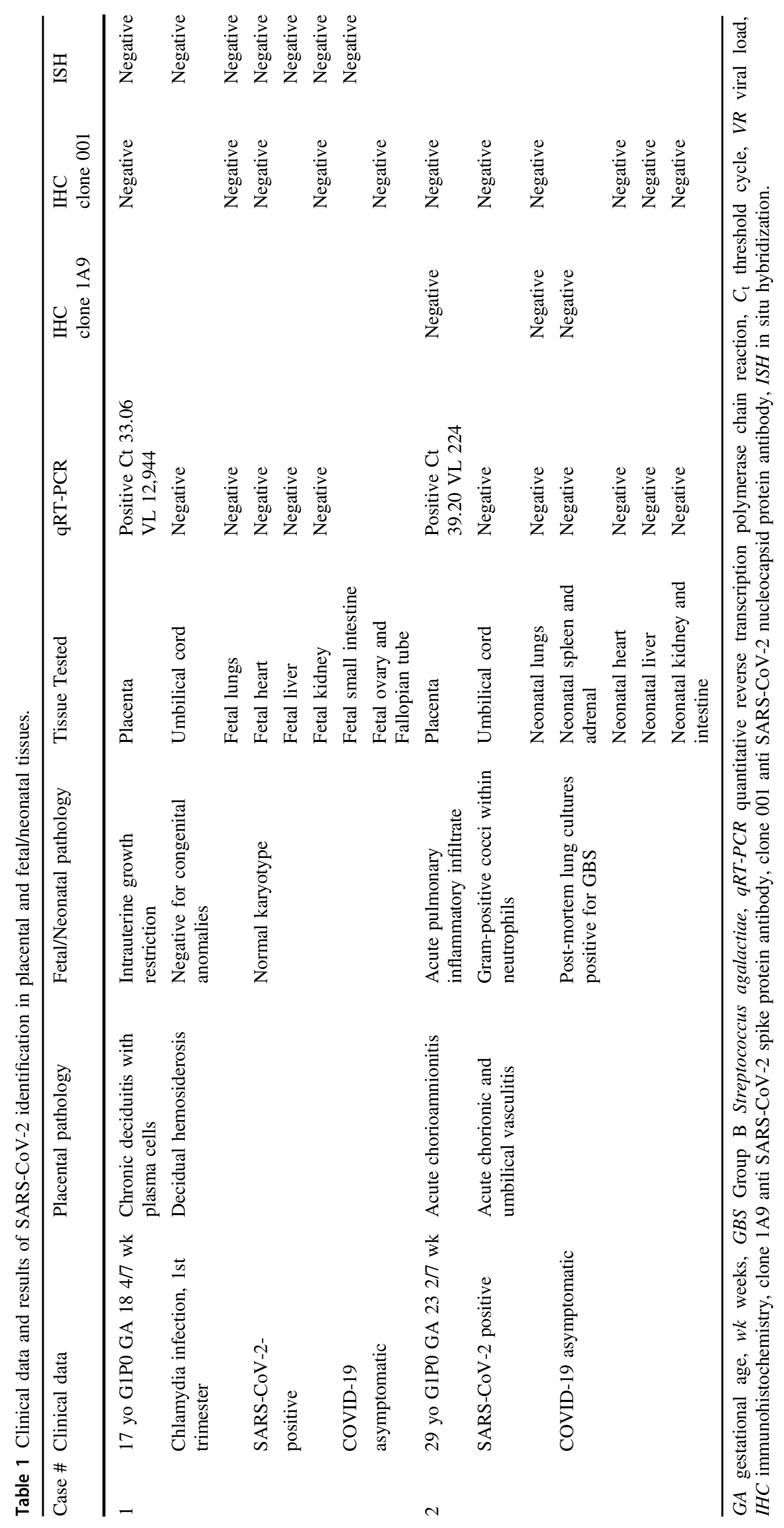


Funding This research received no specific grant from any funding agency in the public, commercial, or not-for-profit sectors.

Author contributions JEV acquired data and wrote the manuscript, AMC conducted experiments and edited the manuscript, ACU designed experiments and edited the manuscript, LD collected material, supervised the study and edited the manuscript.

\section{Compliance with ethical standards}

Conflict of interest The authors declare that they have no conflict of interest.

Publisher's note Springer Nature remains neutral with regard to jurisdictional claims in published maps and institutional affiliations.

\section{References}

1. Kirtsman M, Diambomba Y, Poutanen SM, Malinowski AK, Vlachodimitropoulou E, Parks WT, et al. Probable congenital SARS-CoV-2 infection in a neonate born to a woman with active SARS-CoV-2 infection. CMAJ. 2020;192:E647-50.

2. Vivanti AJ, Vauloup-Fellous C, Prevot S, Zupan V, Suffee S, Do Cao J, et al. Transplacental transmission of SARS-CoV-2 infection. Nat Commun. 2020;11:3572.

3. Hosier H, Farhadian SF, Morotti RA, Deshmukh U, Lu-Culligan A, Campbell KH, et al. SARS-CoV-2 infection of the placenta. J Clin Invest. 2020;130:4947-53.

4. Baud D, Greub G, Favre G, Gengler C, Jaton K, Dubruc E, et al. Second-trimester miscarriage in a pregnant woman with SARSCoV-2 infection. JAMA. 2020;323:2198-200.

5. Penfield CA, Brubaker SG, Limaye MA, Lighter J, Ratner AJ, Thomas KM, et al. Detection of SARS-COV-2 in Placental and Fetal Membrane Samples. Am J Obstet Gynecol MFM. 2020;2:100133. 